(

mappima

\title{
The disco
}

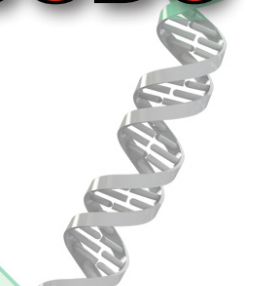

\section{cha} chromosome structure. Jeffrey Perkel takes a closer look into these "chromosome neighborhoods."

Eukar promoters who promoters who are themselves infuenced by the concerted action of distal enhancer and repressor sequences often located hundreds of kilobases away. In short, eukaryotic gene expression is a complex and intricate business.

Cartoons and diagrams usually portray these interactions as simple loops, with transcription factors binding to regulatory sequences, acting as bridges to tweak the activity of distant gene promoters. Yet it's clear that in reality, the nucleus must be loaded with such loops: Mammalian genomes, notes Job Dekker, Professor and Co-director of the Program in Systems Biology at the University of Massachusetts Medical School, are chock full of regulatory sequences, some constitutive, others transmitting subtle developmental cues. Given the genetic cacophony this situation can produce, how can cells limit genetic "distractions" to ensure regulatory sequences act only where they're supposed to?

\section{Discovering the neighborhood}

A few years ago, three independent research teams, one co-directed by Dekker, hit on a potential solution to this question. The teams used chromosome interaction mapping techniques-genome-scale variants of a method Dekker developed called "chromosome conformation cap- ture" (3C) - to trace the chromosomal contacts in the mouse $X$-inactivation center (XIC) (1), mouse and human embryonic stem cells (ESCs) (2), and Drosophila embryos (3). All of these studies arrived at a similar conclusion: metazoan chromosomes tend to organize into a series of discrete topological domains ranging from hundreds of kilobases to a megabase in size. When plotted on heat maps of chromosomal contacts, the domains-termed "topologically associating domains" (TADs)-appear as a series of bright squares along a diagonal representing genomic position, a pattern indicating that these sequences make extensive contacts within their host domains, but rarely outside of them.

According to Edith Heard, Head of the Department of Genetics and Developmental Biology at the Institut Curie in Paris, TAD positioning appears to be more or less stable across developmental stage, evolutionary history, and even transcriptional activity. It was her team that analyzed transcriptome patterns in mouse ESC development, correlating expression dynamics with TADs in the XIC region. Genes within individual TADs, the team found, tended to rise and fall in concert. But transcriptional profiles from adjacent TADs could be diametrically opposed-like independent bubbles of activity -that is, until mutations alter them. When the team examined chromosomal contacts in the XIC of mouse ESCs containing a deletion of one TAD boundary, 
they detected "ectopic contacts" not found in wild-type profiles, accompanied by inappropriate gene expression.

"TADs seem to help keep regions together that maybe need to be coordinately expressed and to separate regions that need to be differently expressed," Heard explains.

Dekker likens TADs to city neighborhoods, thriving genomic communities effectively isolated from one another-and from regulatory signals they might otherwise share. A gene located in one such domain, he says, "doesn't really see most of the genome, it doesn't interact with most of the genome. It only sees its own neighborhood."

Bing Ren, Professor of Cellular and Molecular Medicine at the University of California, San Diego, who led the ESC study (2), calls these domains "the unit of chromosome folding," the genomic equivalent of the alpha helices and beta sheets that give proteins their proper shape.

\section{A TAD of explanation}

Evidence is now accumulating to suggest TADs may underlie previously identified elements of chromosomal organization. In 2008, Bas van Steensel of the Netherlands Cancer Institute discovered and mapped genomic features called lamina-associated domains (LADs). LADs are chromosomal regions associated with the nuclear lamina, the protein matrix underlying the nuclear membrane. In general, genes in LADs are poorly expressed. As ESCs differentiate, Heard found, sequences within the XIC can alter their contacts with the lamina. Yet a given TAD is either lamina-associated or not, and those TAD endpoints remain constant across differentiation states; LADs, she says, "often 'respect' the boundaries of the underlying TAD." David Gilbert, the

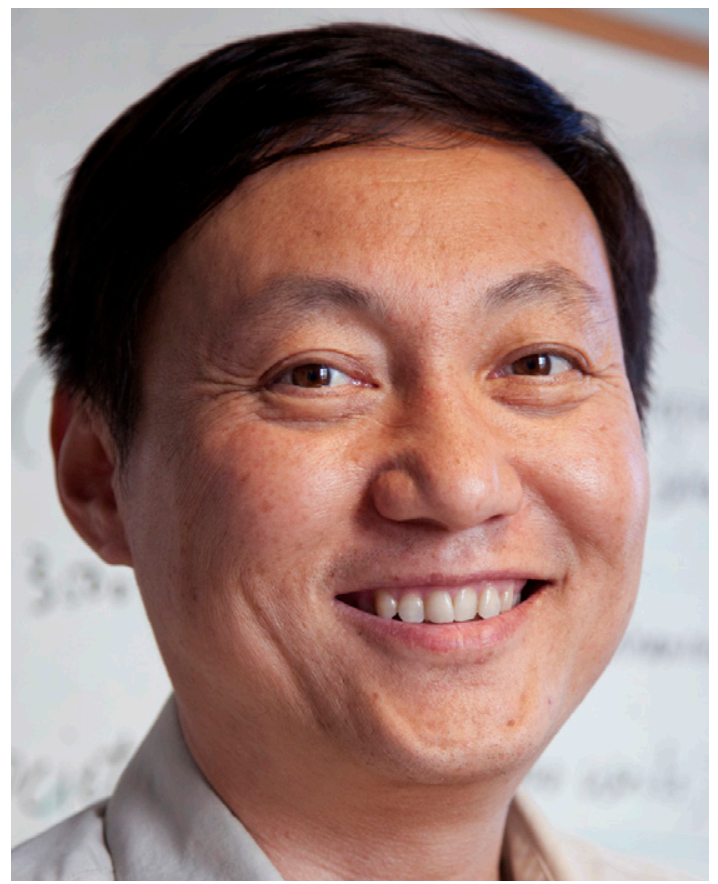

Bing Ren, from University of California, San Diego, has been generating and annotating genome-wide TAD data sets. Credit: Bing Ren.

J. Herbert Taylor Distinguished Professor of Molecular Biology at Florida State University, made a similar observation for replication timing domains, units of chromosomal DNA that undergo coordinate replication during mitosis (7). "There is an almost one-to-one correspondence of TADs to replication domains," he says.

What truly defines a TAD is still unclear. The available data suggest that their boundaries are enriched for highly expressed housekeeping genes and binding sites for proteins such as the transcription factor CTCF (CCCTCbinding factor)-previously implicated in so-called chromosomal "insulators"-and cohesin. The HoxA gene cluster, for instance, comprises 11 genes and at least 2 TADs marked by CTCFrich boundaries. Danny Reinberg at the New York University School of Medicine recently showed that for ESCs the 11 HoxA genes are silent; in motor neurons, 6 contiguous genes on one side of a TAD boundary (Hoxa16) are active. When the team knocked out an intervening CTCF-binding site using CRISPR/Cas-mediated genome editing, the boundary was disrupted, causing chromosomal contacts to extend further into the gene cluster and the next gene in line, Hoxa7, to turn on (4).

In May, Stefan Mundlos of the Max Planck Institute for Molecular Genetics in Berlin and his colleagues published a

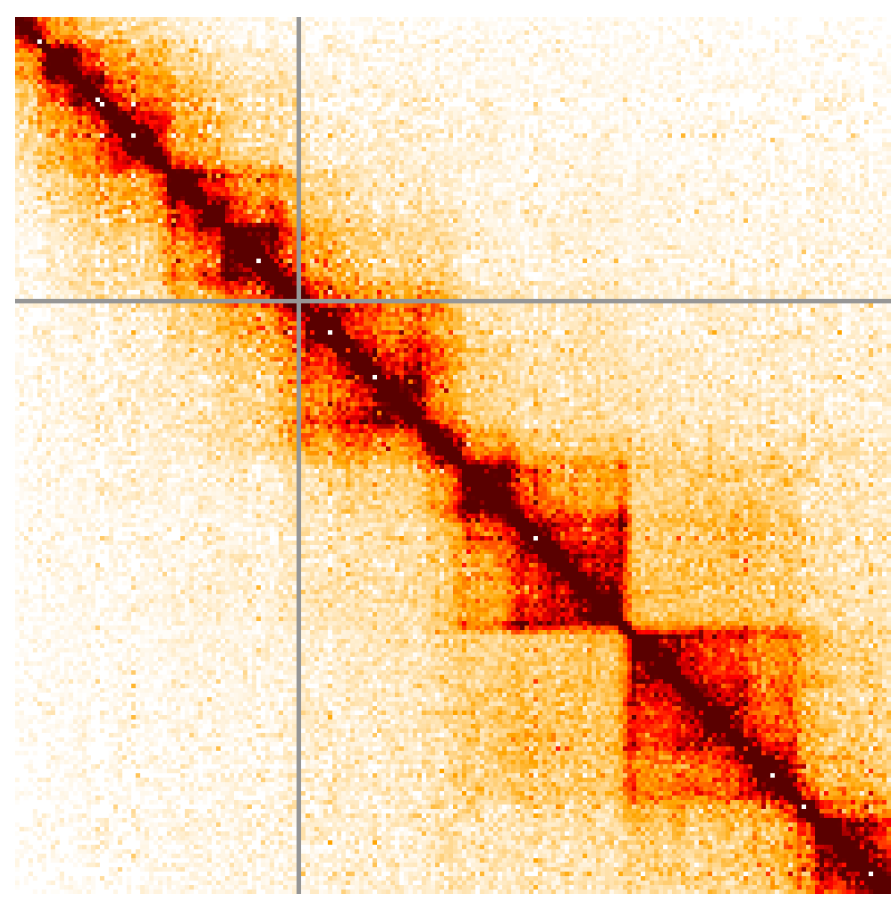

Hi-C data heat maps of TADs from Chromosome 16. Credit: Anne-Laure Valton, Marlies Oomen, and Job Dekker. study underscoring the clinical impact of TAD boundaries. The team found that three congenital conditions, all of which result in malformation of the human hand, stem from chromosomal duplications or deletions altering TAD boundaries near the EPHA4 gene (6). In one such case (brachydactyly), the genomic region including EPHA4 itself is deleted; the two others (F-syndrome and polydactyly) are marked by chromosomal duplications or inversions located upstream. EPHA4 knockout mice have no skeletal defects, the authors note, but EPHA4 is a genetic oasis within a "gene desert," and that desert contains functional regulatory sequences. Chromosomal alterations 


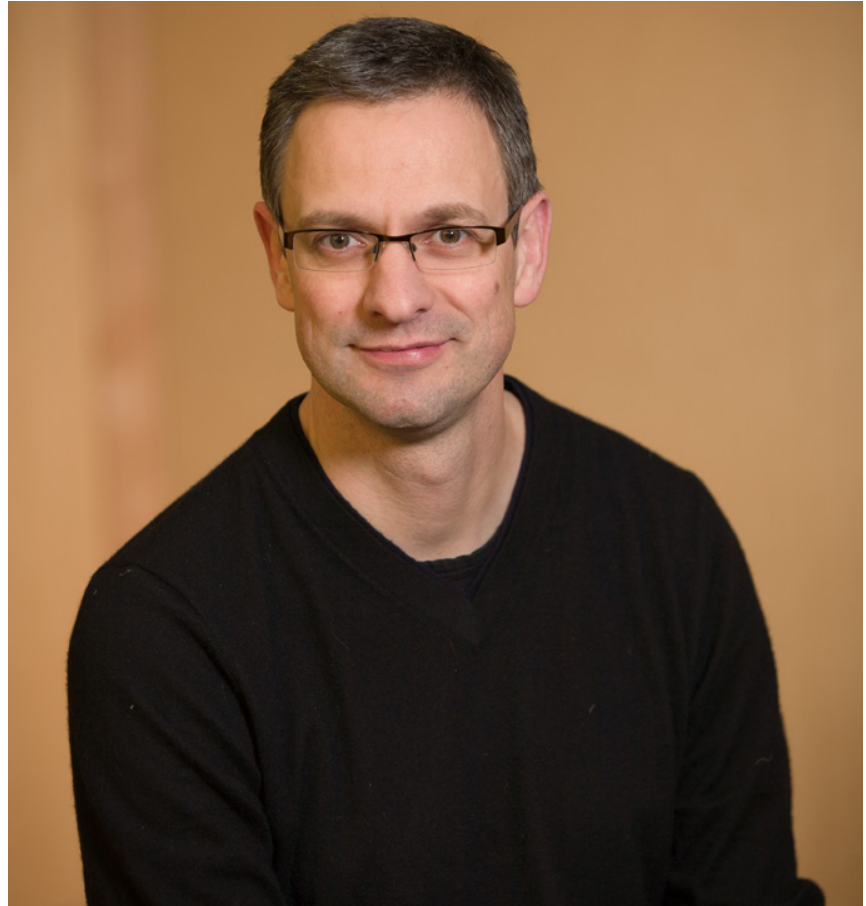

Job Dekker, from the University of Massachusetts Medical School, has worked to develop the tools to define chromosomal interactions. Credit: Job Dekker.

associated with these diseases impact the those sequences' spheres-of-influence, inappropriately activating nearby genes and producing a range of limb malformations.

"They're not actually changing the sequence content in this locus," Heard says, "they're just changing the positioning of the gene within its TAD. And that clearly has an impact on the way it's going to be expressed during development, and leads to a phenotype." This, she adds, "is highly consistent with emerging studies published by the [François] Spitz group at EMBL and others showing that long-range enhancers and regulatory landscapes of complex loci tend indeed to be contained within single TADs."

Ren, who wrote a companion commentary to the study, calls it "one of the best set[s] of evidence that supports the functional importance of TAD structure."

Mundlos says he began investigating the possibility that TADs could explain congenital hand malformation based on a seminar he attended, given by Ren. In searching for a genetic cause of the diseases, his team had identified alterations in chromosomal structure, but was at a loss to explain how they might lead to disease. "I suddenly felt this is how it has to work," he says, calling the seminar an "absolute eye-opener." And he predicts that TAD alterations could figure prominently in other diseases, too, including cancer, that also tend to involve large-scale chromosomal rearrangements. In 2014, Ruud Delwel at Erasmus University Medical Center in the Netherlands and colleagues reported a form of acute myeloid leukemia caused by inversion or translocation of sequences on the long arm of chromosome 3 that disrupt a TAD boundary, thereby dialing down the expression of the GATA2 gene while up-regulating the EVI1 proto-oncogene (5). "It places an enhancer in the same TAD as an oncogene, where it shouldn't be to turn on the oncogene, while removing a regulatory element from a tumor suppressor," Dekker explains. "I mean, you can't make this up."

According to Heard, the discovery of TADs has practical as well as clinical implications. First, they simplify the design and interpretation of genome-wide association studies (GWAS). If researchers need to know where regulatory factors are that control gene expression, they needn't look genome-wide. "You can just probably focus on the one TAD that the gene is located in."

They also should aid in the development of transgenes and gene-therapy vectors. Historically, Heard notes, researchers have been plagued by transgenes that fail to perform as expected because they integrate into unfavorable genomic regions and thus are either over- or under-expressed. But a transgene flanked by intact TAD boundaries would be expected to express the gene as researchers intend. "What I say to people when they come to me for advice on transgenics is, "go and look at the TAD Atlas'," she says, referring to the genomewide TAD data sets generated and annotated independently by the laboratories of Bing Ren and Erez Lieberman Aiden at the Baylor College of Medicine.
Cartoon representation of the interactions that can occur within TADs. Also represented is the barrier that exists between TADs restricting their activities. Credit: Thomas Splettstoesser (www.scistyle.com). 


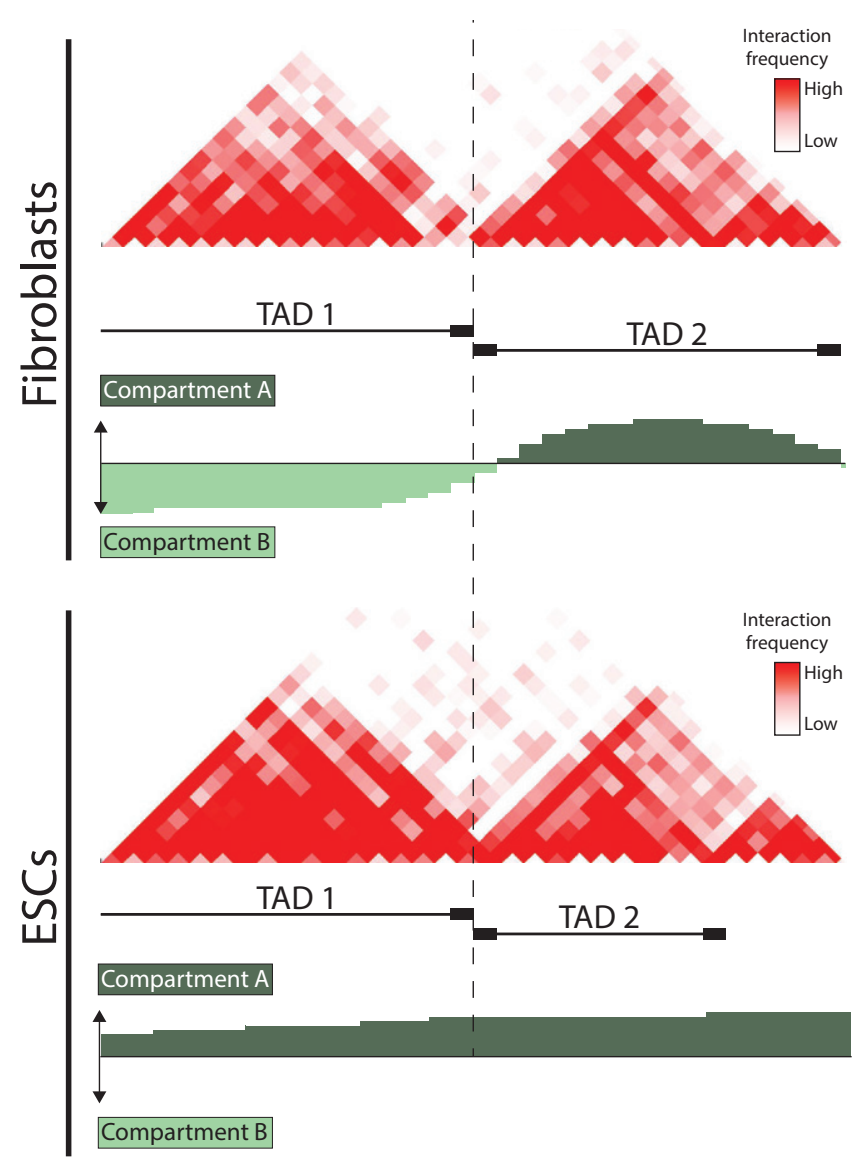

HiC data for two neighboring TADs. Heat maps for IMR90 fibroblasts (top) and human ES cells (bottom) show pairwise interaction frequencies. Dashed line marks the boundary between the two TADs. Credit: David Gorkin.

\section{Lingering questions}

As chromosomal architecture comes into focus, fundamental questions remain. How, for instance, are TADs formed, and how are they maintained? TADs seem to disappear during mitosis, Dekker notes, suggesting they are assembled and disassembled dynamically. Also unclear is how big they can be, and even their very physical nature. "I don't think anybody really knows what they are or what they look like," says Peter Fraser, head of the Nuclear Dynamics Programme at the Babraham Institute in Cambridge, U.K., who has developed a single-cell version of the genomescale chromosome conformation capture approach called $\mathrm{Hi}-\mathrm{C}$, which is commonly used in TAD studies. Researchers, he says, have developed models involving "little mushroomshaped broccoli things where the TAD boundaries interact, and there's a little sort of fluffy domain in the middle that loops out." But Fraser is skeptical. "I don't think any of the explanations really fit all of the data."

For Dekker, the TAD story is just beginning. New interaction atlases offer ever-higher resolution of chromosomal conformation, Dekker notes, and researchers will soon be able to probe within TAD boundaries. "There's all kinds of phenomena happening inside the TAD," Dekker says. "There might be further, smaller squares that we haven't identified yet."

\section{References}

1. Nora, EP, et al., "Spatial partitioning of the regulatory landscape of the X-inactivation centre," Nature, 485:381-5, 2012.

2. Dixon, JR, et al., "Topological domains in mammalian genomes identified by analysis of chromatin interactions," Nature, 485:376-80, 2012

3. Sexon, T, et al., "Three-dimensional folding and functional organization principles of the Drosophila genome," Cell, 148:458-72, 2012.

4. Narendra, $\mathbf{V}$, et al., "CTCF establishes discrete functional chromatin domains at the Hox clusters during differentiation," Science, 347:1017-21, 2015.

5. Gröschel, S, et al., "A single oncogenic enhancer rearrangement causes concomitant EVI1 and GATA2 deregulation in leukemia," Cell, 157:369-81, 2014.

6. Lupiáñez, DG, et al., "Disruptions of topological chromatin domains cause pathogenic rewiring of gene-enhancer interactions," Cell, http://dx.doi. org/10.1016/j.cell.2015.04.004, 2015.

7. Pope, BD, et al, "Topologically associating domains are stable units of replication-timing regulation," Nature, 515:402-5, 2014.

Written by Jeffrey Perkel, Ph.D. 㖥

BioTechniques 58:280-284 (June 2015) doi: 10.2144/000114296 